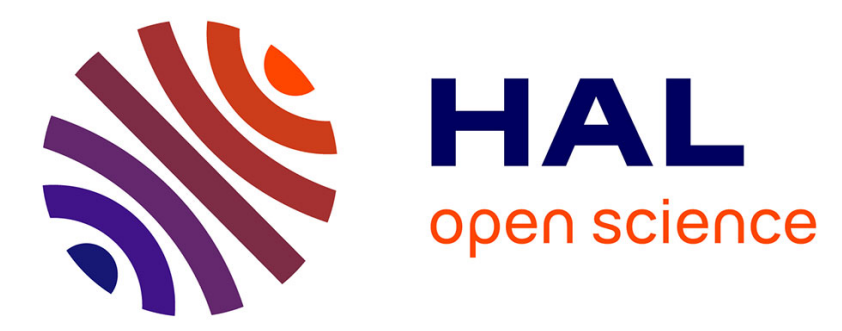

\title{
Permanent genetic resources added to molecular ecology resources database 1 august 2011-30 september 2011
}

S.W. A'Hara, Paul Amouroux, Emily.E. Argo, Arman Avand-Faghih, Ashoktaru Barat, Luiz Barbieri, Theresa M. Bert, R. Blatrix, Aurelie Blin, D. Bouktila, et al.

\section{To cite this version:}

S.W. A'Hara, Paul Amouroux, Emily.E. Argo, Arman Avand-Faghih, Ashoktaru Barat, et al.. Permanent genetic resources added to molecular ecology resources database 1 august 2011-30 september 2011. Molecular Ecology Resources, 2012, 12 (1), pp.185-189. 10.1111/j.1755-0998.2011.03088.x . hal-02653040

\section{HAL Id: hal-02653040 \\ https://hal.inrae.fr/hal-02653040}

Submitted on 29 May 2020

HAL is a multi-disciplinary open access archive for the deposit and dissemination of scientific research documents, whether they are published or not. The documents may come from teaching and research institutions in France or abroad, or from public or private research centers.
L'archive ouverte pluridisciplinaire HAL, est destinée au dépôt et à la diffusion de documents scientifiques de niveau recherche, publiés ou non, émanant des établissements d'enseignement et de recherche français ou étrangers, des laboratoires publics ou privés. 


\section{PERMANENT GENETIC RESOURCES NOTE Permanent Genetic Resources added to Molecular Ecology Resources Database 1 August 2011-30 September 2011} A. AVAND-FAGHIH, ${ }^{6}$ ASHOKTARU BARAT, ${ }^{7}$ LUIZ BARBIERI, ${ }^{8}$ THERESA M. BERT, ${ }^{8}$ R. BLATRIX, $9^{9}$ AURÉLIE BLIN, ${ }^{10}$ D.

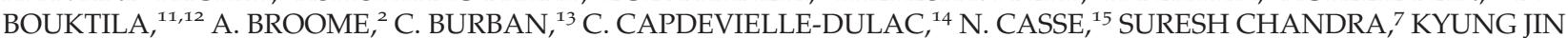
$\mathrm{CHO},{ }^{16} \mathrm{~J}$. E. COTTRELL,${ }^{2}$ CHARLES R. CRAWFORD,${ }^{8}$ MICHELLE C. DAVIS,${ }^{8}$ H. DELATTE,${ }^{17}$ NICOLAS DESNEUX ${ }^{18}$ C. DJIETO-LORDON, ${ }^{19}$ M. P. DUBOIS, ${ }^{9}$ R. A. A. M. EL-MERGAWY,${ }^{20}$ C. GALLARDO-ESCÁRATE, ${ }^{21}$ M. GARCIA,${ }^{9}$ MARY M. GARDINER, ${ }^{22}$ THOMAS GUILLEMAUD, ${ }^{10}$ P. A. HAYE, ${ }^{23}$ B. HELLEMANS, ${ }^{24}$ P. HINRICHSEN, ${ }^{25} \mathrm{JI} \mathrm{HYUN} \mathrm{JEON,}^{26}$

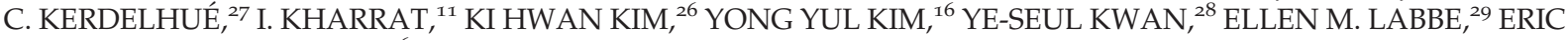

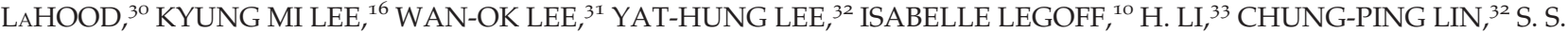
LIU, ${ }^{34}$ Y. G. LIU, ${ }^{35}$ D. LONG $^{36}{ }^{36}$ G. E. MAES,${ }^{24}$ E. MAGNOUX,${ }^{37}$ PRABIN CHANDRA MAHANTA, ${ }^{7}$ H. MAKNI, ${ }^{11,38}$

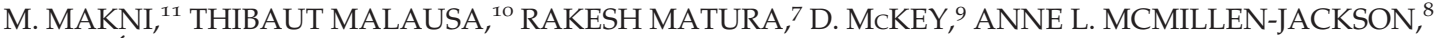

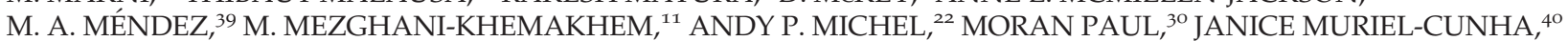
S. NIBOUCHE ${ }^{17}$ F. NORMAND,${ }^{3}$ ERIC P. PALKOVACS,${ }^{5}$ VEENA PANDE,${ }^{41}$ K. PARMENTIER,${ }^{42}$ J. PECCOUD,${ }^{9}$ F. PIATSCHECK, ${ }^{9}$ CECILIA PUCHULUTEGUI, ${ }^{8}$ R. RAMOS, ${ }^{25,43}$ G. RAVEST, ${ }^{25}$ HEINZ RICHNER,${ }^{44}$ J. ROBBENS ${ }^{42}$ D. ROCHAT, ${ }^{45}$ J. ROUSSELET, ${ }^{37}$ VERENA SALADIN, ${ }^{44}$ M. SAUVE, ${ }^{9}$ ORA SCHLEI, ${ }^{6}{ }^{6}$ THOMAS F. SCHULTZ, ${ }^{5}$ A. R. SCOBIE, ${ }^{47}$

N. I. SEGOVIA, ${ }^{23}$ SEIFU SEYOUM, ${ }^{8}$ J.-F. SILVAIN, ${ }^{14}$ ELISABETH TABONE, ${ }^{48}$ J. K. J. VAN HOUDT, ${ }^{24,49}$

S. G. VANDAMME, ${ }^{42,24}$ F. A. M. VOLCKAERT, ${ }^{24}$ JOHN WENBURG, ${ }^{46}$ THEODORE V. WILLIS, ${ }^{50}$ YONG-JIN WON, ${ }^{28}$ N. H. YE, ${ }^{51}$ W. ZHANG ${ }^{22}$ and Y. X. ZHANG ${ }^{35}$

${ }^{1}$ Molecular Ecology Resources Editorial Office, 6270 University Blvd, Vancouver, British Columbia, Canada V6T 1Z4, ${ }^{2}$ Forest Research, Northern Research Station, Roslin, Midlothian, Scotland EH25 9SY, UK, ${ }^{3}$ CIRAD, UPR HortSys, Station de Bassin Plat, BP180, F-97455 Saint-Pierre, La Réunion, France, ${ }^{4}$ Université de la Réunion, 15 avenue René Cassin BP 7151, F-97715 Saint-Denis Messag, Cedex 9, La Réunion, France, ${ }^{5}$ Marine Conservation Molecular Facility, Duke University Marine Laboratory, Nicholas School of the Environment, 135 Duke Marine Lab Road, Beaufort, NC 28516, USA, ${ }^{6}$ Plant Pests E Diseases Research Institute, PO box 1454, 19395 Tehran, Iran, ${ }^{7}$ Molecular Genetics Laboratory, Directorate of Coldwater Fisheries Research, Indian Council of Agricultural Research, Bhimtal-263136, Nainital, Uttarakhand, India, ${ }^{8}$ Florida Fish and Wildife Research Institute, 100 Eighth Avenue S.E., Saint Petersburg, FL 33701-5095, USA, ${ }^{9}$ Centre d'Ecologie Fonctionnelle et Evolutive (CEFE), UMR 5175 (CNRS, Université Montpellier 2), 1919 route de Mende, 34293 Montpellier Cedex 5, France, ${ }^{10}$ Equipe 'Biologie des populations en interaction', UMR 1301 IBSV INRA-CNRS-Université de Nice-Sophia Antipolis 400 route des Chappes, 06903 Sophia-Antipolis Cedex, France,

${ }^{11}$ Unité génomique des insectes ravageurs des cultures d'intérêt agronomique, Faculté des Sciences de Tunis, Université de Tunis-ElManar, Tunisia, ${ }^{12}$ Institut Supérieur de Biotechnologie Béja, Université de Jendouba, Tunisia, ${ }^{13}$ INRA, UMR1202 BIOGECO (INRA/Université de Bordeaux), F-33610 Cestas, France, ${ }^{14}$ IRD, UR 072, Laboratoire Evolution, Génomes et Spéciation, UPR 9034, CNRS, 91198 Gif-sur-Yvette, France and Université Paris Sud 11, 91405 Orsay Cedex, France, ${ }^{15}$ Laboratoire Mer, Molécules, Santé (MMS), Université du Maine, Le Mans, France, ${ }^{16}$ Division of Seed E Seedling Management, Korea Forest Seed and Variety Center, 72, Suhoeri-ro, Chungju-si, Chungcheongbuk-do, 380-941, Korea, ${ }^{17}$ CIRAD, UMR PVBMT, 7 chemin de l'IRAT, Ligne Paradis, F-97410 Saint-Pierre, La Réunion, France, ${ }^{18}$ Unité de recherche intégrée en horticulture, INRA, 400 route des Chappes, 06903 Sophia-Antipolis Cedex, France, ${ }^{19}$ Laboratory of Zoology, University of Yaoundé I, Faculty of Science, PO Box 812, Yaoundé, Cameroun, ${ }^{20}$ Department of Molecular Biology, Genetic Engineering and Biotechnology Research Institute (GEBRI), Minoufia University, El-Sadat City, Minoufia, Egypt, ${ }^{21}$ Departamento de Oceanografía, Facultad de Ciencias Naturales y Oceanográficas, Centro de Biotecnología, Universidad de Concepción, Casilla 160-C, Concepción, Chile, ${ }^{22}$ Department of Entomology, The Ohio Agricultural Research and Development Center, The Ohio State University, 1680 Madison Avenue, Wooster, OH 44691, USA, ${ }^{23}$ Departamento de Biología Marina, Universidad Católica del Norte E Centro de Estudios Avanzados en Zonas Áridas (CEAZA), Larrondo 1281, Coquimbo, Chile, ${ }^{24}$ Katholieke Universiteit Leuven (KULeuven), Laboratory of Animal Diversity and Systematics, BioGenomics Division, Charles Deberiotstraat 32, 3000 Leuven, Belgium, ${ }^{25}$ Laboratorio de Biotecnología, Centro de Investigación La Platina, Instituto de Investigaciones Agropecuarias, INIA, Santa Rosa 11,610, P.O. Box 439-3, Santiago, Chile, ${ }^{26}$ Biomedic, 1143-3, Joongdong, Wonmi-gu, Bucheon-si, Gyeonggi-do, 420-020, Korea, ${ }^{27}$ INRA, UMR CBGP (INRA/IRD/CIRAD/Montpellier Supagro), F34988 Montferrier-sur-Lez, France, ${ }^{28}$ Division of EcoScience, Ewha Womans University, Seoul, Korea, ${ }^{29}$ Department of Biology, University of Southern Maine, 96 Falmouth St, Portland, ME 04102, USA, ${ }^{30}$ Conservation Biology Division, Northwest Fisheries Science Center, 2725 Montlake East, Seattle, WA 98112, USA, ${ }^{31}$ Inland Fisheries Research Institute, National Fisheries Research E Development Institute, Gapyeong, Gyeonggi-do, Korea, ${ }^{32}$ Department of Life Science E Center for Tropical Ecology and Biodiversity, Tunghai University, Taichung, 40704, Taiwan, ${ }^{33}$ Jiangsu Provincial Key Laboratory of Coastal Wetland Bioresources and Environ- 
mental Protection, Yancheng Teachers University, Yancheng 224002, China, ${ }^{34}$ Ocean University of China, Qingdao 266003, China, ${ }^{35}$ Shandong Entry-Exit Inspection and Quarantine Bureau, Qingdao 266002, China, ${ }^{36}$ Plantlife Scotland, Balallan House, Allan Park, Stirling, Scotland FK8 2QG, UK, ${ }^{37}$ INRA, UR633 Zoologie forestière, 45075 Orléans cedex 2, France, ${ }^{38}$ Institut Supérieur de l'Animation pour la Jeunesse et la Culture, Bir El Bey, Université de Tunis, Tunisia, ${ }^{39}$ Laboratorio de Genética y Evolución, Facultad de Ciencias, Univ. de Chile, Las Palmeras 3425, Nuñoa, Box 780-0024, Santiago, Chile, ${ }^{40}$ Universidade Federal do Pará - Altamira, Faculdade de Ciências Biológicas, Rua Coronel José Porfírio, N 2515, 68372-040 - Altamira, PA, Brasil, ${ }^{41}$ Department of Biotechnology, Kumaon University, Bhimtal-263136, Uttarakhand, India, ${ }^{42}$ Institute for Agricultural and Fisheries Research (ILVO-Fisheries), Ankerstraat 1, 8400 Ostend, Belgium, ${ }^{43}$ Syngenta-Chile, Av. Vitacura 2939 Of. 201, Santiago, Chile, ${ }^{44}$ University of Bern, Institute of Ecology and Evolution, Dept. Evolutionary Ecology, Baltzerstrasse 6, 3012 Bern, Switzerland, ${ }^{45}$ UMR 1272, UPMC-INRA, Physiologie de l'insecte: Signalisation et Communication, Route de Saint Cyr, 78026 Versailles Cedex, France, ${ }^{46}$ Conservation Genetics Laboratory, USFWS, 1011 East Tudor Rd., Anchorage, AK 99503, USA, ${ }^{47}$ Cairngorms Rare Plants Project, Scottish Natural Heritage, Achantoul, Aviemore, Inverness-shire, PH22 1QD, ${ }^{48}$ Unité expérimentale de lutte biologique, INRA, 400 route des Chappes, 06903 Sophia-Antipolis Cedex, France, ${ }^{49}$ KULeuven, Laboratory for Cytogenetics and Genome Research, OEN, Herestraat 49, 3000 Leuven, Belgium, ${ }^{50}$ Department of Environmental Science, University of Southern Maine, 37 College Ave, Gorham, ME 04038, USA, ${ }^{51}$ Yellow Sea Fisheries Research Institute, Chinese Academy of Fishery Sciences, Qingdao, 266071, China

\begin{abstract}
This article documents the addition of 299 microsatellite marker loci and nine pairs of single-nucleotide polymorphism (SNP) EPIC primers to the Molecular Ecology Resources (MER) Database. Loci were developed for the following species: Alosa pseudoharengus, Alosa aestivalis, Aphis spiraecola, Argopecten purpuratus, Coreoleuciscus splendidus, Garra gotyla, Hippodamia convergens, Linnaea borealis, Menippe mercenaria, Menippe adina, Parus major, Pinus densiflora, Portunus trituberculatus, Procontarinia mangiferae, Rhynchophorus ferrugineus, Schizothorax richardsonii, Scophthalmus rhombus, Tetraponera aethiops, Thaumetopoea pityocampa, Tuta absoluta and Ugni molinae. These loci were cross-tested on the following species: Barilius bendelisis, Chiromantes haematocheir, Eriocheir sinensis, Eucalyptus camaldulensis, Eucalyptus cladocalix, Eucalyptus globulus, Garra litaninsis vishwanath, Garra para lissorhynchus, Guindilla trinervis, Hemigrapsus sanguineus, Luma chequen. Guayaba, Myrceugenia colchagüensis, Myrceugenia correifolia, Myrceugenia exsucca, Parasesarma plicatum, Parus major, Portunus pelagicus, Psidium guayaba, Schizothorax richardsonii, Scophthalmus maximus, Tetraponera latifrons, Thaumetopoea bonjeani, Thaumetopoea ispartensis, Thaumetopoea libanotica, Thaumetopoea pinivora, Thaumetopoea pityocampa ena clade, Thaumetopoea solitaria, Thaumetopoea wilkinsoni and Tor putitora. This article also documents the addition of nine EPIC primer pairs for Euphaea decorata, Euphaea formosa, Euphaea ornata and Euphaea yayeyamana.
\end{abstract}

This article documents the addition of 299 microsatellite marker loci and nine pairs of single-nucleotide polymorphism (SNP) genotyping primers to the Molecular Ecology Resources Database. Table 1 contains information on the focal species, the number of loci developed, any other species the loci were tested in and the accession numbers for the loci in both the Molecular Ecology Resources Database and GenBank. The authors responsible for each set of loci are listed in the final column. Table 2 presents information on SNP genotyping resources added to the MER database and presents data on the focal species, the number of sequencing primer pairs, the observed number of SNPs, other species the loci were tested in, and the number of allele specific primers or probes. The MER database and GenBank accession numbers and the authors responsible are also listed. Table 3 outlines additional permanent genetic resources that have been uploaded to the MER program wiki (http://tomato.biol. trinity.edu/programs/). A full description of the development protocol for the loci presented in Tables 1 \& 2 can be found on the Molecular Ecology Resources Database (http:/ / tomato.biol.trinity.edu/).

Table 1 Information on the focal species, the number of loci developed, any other species the loci were tested in and the accession numbers for the loci in both the Molecular Ecology Resources (MER) Database and GenBank. The authors responsible for each set of loci are listed in the final column

\begin{tabular}{llllll}
\hline Species & $\begin{array}{l}\text { No. primers } \\
\text { developed }\end{array}$ & $\begin{array}{l}\text { Other species } \\
\text { tested }\end{array}$ & $\begin{array}{l}\text { MER } \\
\text { database no. }\end{array}$ & GenBank accession no. & Authors \\
\hline $\begin{array}{c}\text { Alosa pseudoharengus, } \\
\text { Alosa aestivalis }\end{array}$ & 18 & $\mathrm{n} / \mathrm{a}$ & $47166-47201$ & JN383992-JN384009 & $\begin{array}{c}\text { Labbe, Ellen M.; Argo, Emily E.; } \\
\text { Schultz, Thomas F.; Palkovacs, } \\
\text { Eric P.;Willis, Theodore V. }\end{array}$ \\
\hline
\end{tabular}

Correspondence: Molecular Ecology Resources Primer Development Consortium, E-mail: editorial.office@molecol.com 
Table 1 (Continued)

\begin{tabular}{|c|c|c|c|c|c|}
\hline Species & $\begin{array}{l}\text { No. primers } \\
\text { developed }\end{array}$ & $\begin{array}{l}\text { Other species } \\
\text { tested }\end{array}$ & $\begin{array}{l}\text { MER } \\
\text { database no. }\end{array}$ & GenBank accession no. & Authors \\
\hline Aphis spiraecola & 9 & $\mathrm{n} / \mathrm{a}$ & $47081-47089$ & $\begin{array}{l}\text { HM854169-HM854171, } \\
\text { JN214382-JN214384, } \\
\text { JN214386-JN214388 }\end{array}$ & $\begin{array}{l}\text { Mezghani-Khemakhem, M.; } \\
\text { Kharrat, I.; } \\
\text { Casse, N.; } \\
\text { Bouktila, D.; } \\
\text { Makni, M.; } \\
\text { Makni H. }\end{array}$ \\
\hline $\begin{array}{l}\text { Argopecten } \\
\text { purpuratus }\end{array}$ & 8 & $\mathrm{n} / \mathrm{a}$ & $47373-47380$ & JN674552-JN674559 & $\begin{array}{l}\text { Haye, P. A.; } \\
\text { Segovia, N. I.; } \\
\text { Gallardo-Escárate, C. }\end{array}$ \\
\hline $\begin{array}{l}\text { Coreoleuciscus } \\
\text { splendidus }\end{array}$ & 13 & $\mathrm{n} / \mathrm{a}$ & $47143-47155$ & JF972368-JF972380 & $\begin{array}{l}\text { Kwan, Ye-Seul; } \\
\text { Lee, Wan-Ok; } \\
\text { Won, Yong-Jin }\end{array}$ \\
\hline Garra gotyla & 28 & $\begin{array}{l}\text { G. para } \\
\text { lissorhynchus, } \\
\text { G. litaninsis } \\
\text { vishwanath, } \\
\text { Barilius } \\
\text { bendelisis, } \\
\text { Schizothorax } \\
\text { richardsonii, } \\
\text { Tor putitora }\end{array}$ & $47345-47372$ & $\begin{array}{l}\text { HQ288484, HQ288485, } \\
\text { HQ288489-HQ288499, } \\
\text { HQ288501, HQ288502, } \\
\text { HQ288504, HQ288506, } \\
\text { HQ288507, HQ288510, } \\
\text { HQ288511, HQ288517, } \\
\text { HQ288526, HQ288661, } \\
\text { JF268657, JF268662, } \\
\text { JF268664, JF268665 }\end{array}$ & $\begin{array}{l}\text { Matura, Rakesh; } \\
\text { Chandra, Suresh; } \\
\text { Barat, Ashoktaru; } \\
\text { Pande, Veena; } \\
\text { Mahanta, Prabin Chandra }\end{array}$ \\
\hline $\begin{array}{r}\text { Hippodamia } \\
\text { convergens }\end{array}$ & 12 & $\mathrm{n} / \mathrm{a}$ & $47397-47408$ & JN565049-JN565060 & $\begin{array}{l}\text { Michel, Andy P.; } \\
\text { Zhang, W.; } \\
\text { Gardiner, Mary M. }\end{array}$ \\
\hline Linnaea borealis & 10 & $\mathrm{n} / \mathrm{a}$ & $47156-47165$ & JN674504-JN674512 & $\begin{array}{l}\text { A'Hara, S. W.; } \\
\text { Scobie, A. R.; } \\
\text { Broome, A.; } \\
\text { Long, D.; } \\
\text { Cottrell, J. E. }\end{array}$ \\
\hline $\begin{array}{l}\text { Menippe } \\
\text { mercenaria, } \\
\text { M. adina }\end{array}$ & 22 & $\mathrm{n} / \mathrm{a}$ & $46925-46968$ & GU970048-GU970069 & $\begin{array}{l}\text { Seyoum, Seifu; } \\
\text { Bert, Theresa M.; } \\
\text { Puchulutegui, Cecilia; } \\
\text { Davis, Michelle C.; } \\
\text { Muriel-Cunha, Janice; } \\
\text { Crawford, Charles R.; } \\
\text { Mcmillen-Jackson, Anne L.; } \\
\text { Barbieri, Luiz }\end{array}$ \\
\hline Parus major & 15 & $\mathrm{n} / \mathrm{a}$ & $47128-47142$ & HQ263118-HQ263132 & $\begin{array}{l}\text { Saladin, Verena; } \\
\text { Richner, Heinz }\end{array}$ \\
\hline Pinus densiflora & 16 & $\mathrm{n} / \mathrm{a}$ & $47381-47396$ & JN634766-JN634781 & $\begin{array}{l}\text { Lee, Kyung Mi; } \\
\text { Kim, Yong Yul; } \\
\text { Kim, Ki Hwan; } \\
\text { Jeon, Ji Hyun; } \\
\text { Cho, Kyung Jin }\end{array}$ \\
\hline $\begin{array}{l}\text { Portunus } \\
\text { trituberculatus }\end{array}$ & 11 & $\begin{array}{l}\text { P. pelagicus, } \\
\text { Eriocheir } \\
\text { sinensis, } \\
\text { Hemigrapsus } \\
\text { sanguineus, } \\
\text { Chiromantes } \\
\text { haematocheir, } \\
\text { Parasesarma } \\
\text { plicatum }\end{array}$ & $46914-46924$ & JF505633-JF505643 & $\begin{array}{l}\text { Li, H.; } \\
\text { Ye, N. H.; } \\
\text { Liu, Y. G.; } \\
\text { Zhang, Y. X.; } \\
\text { Liu, S. S. }\end{array}$ \\
\hline
\end{tabular}


188 PERMANENT GENETIC RESOURCES NOTE

Table 1 (Continued)

\begin{tabular}{|c|c|c|c|c|c|}
\hline Species & $\begin{array}{l}\text { No. primers } \\
\text { developed }\end{array}$ & $\begin{array}{l}\text { Other species } \\
\text { tested }\end{array}$ & $\begin{array}{l}\text { MER } \\
\text { database no. }\end{array}$ & GenBank accession no. & Authors \\
\hline $\begin{array}{l}\text { Procontarinia } \\
\text { mangiferae }\end{array}$ & 11 & $\mathrm{n} / \mathrm{a}$ & $47057-47067$ & JF746879-JF746889 & $\begin{array}{l}\text { Amouroux, P.; } \\
\text { Normand, F.; } \\
\text { Nibouche, S.; } \\
\text { Delatte H. }\end{array}$ \\
\hline $\begin{array}{l}\text { Rhynchophorus } \\
\text { ferrugineus }\end{array}$ & 15 & $\mathrm{n} / \mathrm{a}$ & $47113-47127$ & JN374673-JN374687 & $\begin{array}{l}\text { Capdevielle-Dulac, C.; } \\
\text { El-Mergawy, R. A. A. M.; } \\
\text { Avand-Faghih, A.; } \\
\text { Rochat, D.; } \\
\text { Silvain, J.-F. }\end{array}$ \\
\hline $\begin{array}{l}\text { Schizothorax } \\
\text { richardsonii }\end{array}$ & 34 & $\mathrm{n} / \mathrm{a}$ & $47292-47325$ & $\begin{array}{l}\text { HM591233-HM591236, } \\
\text { HM591238, } \\
\text { HM591240-HM591242, } \\
\text { HM591244, } \\
\text { HM591246-HM591256, } \\
\text { HM591258, HM591260, } \\
\text { HM591264-HM591266, } \\
\text { HM591270-HM591272, } \\
\text { HM591276, HM591278, } \\
\text { HM591279, HM591281, } \\
\text { HM591283 }\end{array}$ & $\begin{array}{l}\text { Barat, Ashoktaru; } \\
\text { Chandra, Suresh; } \\
\text { Matura, Rakesh }\end{array}$ \\
\hline $\begin{array}{l}\text { Scophthalmus } \\
\text { rhombus }\end{array}$ & 15 & S. maximus & 47090-47104 & JF900344-JF900358 & $\begin{array}{l}\text { Vandamme, S. G.; } \\
\text { Maes, G. E.; } \\
\text { Van Houdt, J. K. J.; } \\
\text { Hellemans, B.; } \\
\text { Robbens, J.; } \\
\text { Parmentier, K.; } \\
\text { Volckaert, F. A. M. }\end{array}$ \\
\hline $\begin{array}{l}\text { Tetraponera } \\
\text { aethiops }\end{array}$ & 14 & T. latifrons & $46982-47009$ & JN190035-JN190048 & $\begin{array}{l}\text { Piatscheck, F.; } \\
\text { Djieto-Lordon, C.; } \\
\text { Garcia, M.; } \\
\text { Sauve, M.; } \\
\text { Peccoud, J.; } \\
\text { Dubois, M. P.; } \\
\text { McKey, D.; } \\
\text { Blatrix, R. }\end{array}$ \\
\hline $\begin{array}{c}\text { Thaumetopoea } \\
\text { pityocampa }\end{array}$ & 13 & $\begin{array}{l}\text { T. p. ena clade, } \\
\text { T. wilkinsoni, } \\
\text { T. pinivora, } \\
\text { T. libanotica, } \\
\text { T. bonjeani, } \\
\text { T. ispartensis, } \\
\text { T. solitaria }\end{array}$ & 46969-46981 & JN400258-JN400270 & $\begin{array}{l}\text { Burban, C.; } \\
\text { Magnoux, E.; } \\
\text { Rousselet, J.; } \\
\text { Kerdelhué, C. }\end{array}$ \\
\hline Tuta absoluta & 19 & $\mathrm{n} / \mathrm{a}$ & $47326-47344$ & JN680765-JN680783 & $\begin{array}{l}\text { Guillemaud, Thomas; } \\
\text { Legoff, Isabelle; } \\
\text { Blin, Aurélie; } \\
\text { Tabone, Elisabeth; } \\
\text { Desneux, Nicolas; } \\
\text { Malausa, Thibaut }\end{array}$ \\
\hline
\end{tabular}


Table 1 (Continued)

\begin{tabular}{|c|c|c|c|c|c|}
\hline Species & $\begin{array}{l}\text { No. primers } \\
\text { developed }\end{array}$ & $\begin{array}{l}\text { Other species } \\
\text { tested }\end{array}$ & $\begin{array}{l}\text { MER } \\
\text { database no. }\end{array}$ & GenBank accession no. & Authors \\
\hline Ugni molinae & 16 & $\begin{array}{l}\text { Myrceugenia correifolia, } \\
\text { M. colchagüensis, } \\
\text { M. exsucca, } \\
\text { Guindilla trinervis, } \\
\text { Luma chequen. } \\
\text { Guayaba, } \\
\text { Psidium guayaba, } \\
\text { Eucalyptus cladocalix, } \\
\text { E. camaldulensis, } \\
\text { E. globulus }\end{array}$ & $46809-46824$ & HQ917086-HQ917101 & $\begin{array}{l}\text { Ramos, R.; } \\
\text { Ravest, G.; } \\
\text { Méndez, M.A.; } \\
\text { Hinrichsen, P. }\end{array}$ \\
\hline
\end{tabular}

Table 2 Information on the focal species, the sequencing primer pairs developed, the number of single-nucleotide polymorphisms (SNPs) observed and any other species the loci were tested in. The next columns contain the number of allele specific primers and probes developed and the Molecular Ecology Resources (MER) database and GenBank accession numbers, respectively. The authors responsible for each set of loci are listed in the final column

\begin{tabular}{|c|c|c|c|c|c|c|c|c|}
\hline Species & $\begin{array}{l}\text { No. } \\
\text { primer } \\
\text { pairs }\end{array}$ & $\begin{array}{l}\text { No. SNPs } \\
\text { in } \\
\text { sequence }\end{array}$ & $\begin{array}{l}\text { Other } \\
\text { species } \\
\text { tested }\end{array}$ & $\begin{array}{l}\text { No. Allele } \\
\text { specific } \\
\text { primers/ } \\
\text { probe }\end{array}$ & Target gene(s) & $\begin{array}{l}\text { MER } \\
\text { database } \\
\text { numbers }\end{array}$ & $\begin{array}{l}\text { Genbank } \\
\text { Accession no. }\end{array}$ & Authors \\
\hline $\begin{array}{l}\text { Euphaea formosa, } \\
\text { E. yayeyamana, } \\
\text { E. ornata, } \\
\text { E. decorata }\end{array}$ & 9 & $\begin{array}{l}\text { See Table } \\
2 \text { in text } \\
\text { for details. }\end{array}$ & $\mathrm{n} / \mathrm{a}$ & $\mathrm{n} / \mathrm{a}$ & $\begin{array}{l}\text { See Table } 1 \text { in } \\
\text { text for details. }\end{array}$ & $47048-47056$ & $\begin{array}{l}\text { JN246927-JN247002, } \\
\text { JN389796-JN390424 }\end{array}$ & $\begin{array}{l}\text { Lee, Yat-Hung; } \\
\text { Lin, Chung-Ping }\end{array}$ \\
\hline
\end{tabular}

Table 3 Information on other resources recently uploaded to the Molecular Ecology Resources program wiki (http:// tomato.biol.trinity.edu/programs/). The authors are listed in the final column

\begin{tabular}{|c|c|c|c|}
\hline Species & Category & Type of resource & Authors \\
\hline $\begin{array}{c}\text { Oncorhynchus } \\
\text { tshawytscha }\end{array}$ & Technique & $\begin{array}{l}\text { Microsatellite } \\
\text { allele ladder-based } \\
\text { standardization }\end{array}$ & $\begin{array}{l}\text { LaHood, Eric; } \\
\text { Schlei, Ora; } \\
\text { Wenburg, John; } \\
\text { Moran, Paul }\end{array}$ \\
\hline
\end{tabular}

